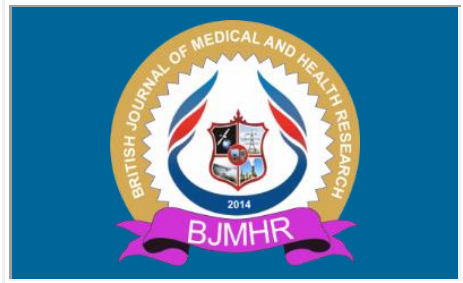

BJMHR

British Journal of Medical and Health Research

Journal home page: www.bjmhr.com

\title{
Dental Health Concerns in Pregnancy: Need for a Collaborative Management Approach
}

\author{
Eram Perwez $^{1 *}$, Shabina Sachdeva ${ }^{1}$, Ateeba Khan ${ }^{2}$, Rizwana Mallick ${ }^{1}$ \\ 1.Department of Prosthodontics, Faculty of Dentistry, JMI, New Delhi
}

\begin{abstract}
A woman undergoes a series of physiological states such as puberty, pregnancy and menarche. A number of anatomical, physiological and hormonal changes occur in pregnancy which not only affects the maternal health but also the health of the child. Oral health is an integral part of overall general health and is often neglected due to lack of knowledge and awareness and an unfavourable attitude. It definitely has its impact upon the systemic health and everyone should be aware about the same. This further needs due consideration in a pregnant women as peculiar oral changes and their impact on the new born infant and pregnancy outcome have been discussed and documented with sufficient evidence. Much has been talked about common oral health issues during pregnancy like gingivitis, periodontitis, pyogenic granuloma etc and their management. Patient's education regarding the oral hygiene and abnormal pregnancy outcome (pre-term birth, low body weight birth) is extremely essential. These conditions may be prevented by appropriate monitoring of dental health in pregnancy and also by providing dental care in such patients. Patients should be referred to the dental health care provider for dental needs during the time of pregnancy. A collaboration of dental and medical health care givers must be made and is an effective channel to provide the best possible treatment and management of these patients. This article discusses the importance of oral health care during pregnancy and how an integration between the medical and dental health provider can go a long way to attend the oral health concerns during this time.
\end{abstract}

Keywords: Gingivitis, oral health, periodontitis, pregnancy, pre-natal, pyogenic granuloma, collaborative 


\section{INTRODUCTION}

Oral health is an integral part of overall general health which is both physical and mental well being. ${ }^{1}$ Pregnancy is a physiological state which brings about a number of oral changes along with other physiological changes affecting other systems in the body. ${ }^{2}$ It is not that pregnancy per se brings these changes but the altered level of hormones during this phase has effects on the already existing oral conditions and in most cases there is a tendency for an exaggerated response. Commonly seen oral changes during pregnancy are gingival hyperplasia, gingivitis, pyogenic granulomas and various salivary alterations (in $\mathrm{pH}$, flow rate, buffering capacity). ${ }^{3}$ Tooth erosion is also seen probably because of the expulsion of gastric content containing gastric acid during morning sickness. ${ }^{4}$ Not just pre-natal, but there is significant effect of oral health status and hygiene of mother on the child's post-natal time, as it is proved that there is high risk of developing early childhood caries (such as rampant caries and nursing bottle caries) in children with mothers having high numbers of cariogenic bacteria. ${ }^{5}$ Oral health promotion, disease prevention, early detection and timely intervention are crucial aspects for maternal and child oral health. ${ }^{6}$ Several medical and dental organizations like American Dental Association (ADA), the American Academy of Periodontology and the American Academy of Paediatric Dentistry have issued guidelines to improve oral health in pregnant women and young children. ${ }^{7}$

\section{COMMON ORAL PROBLEMS IN PREGNANCY}

Pregnancy is accompanied by hormonal changes which tends to affect all the systems of the body including the oral cavity. ${ }^{8}$ Much has been written and discussed about the oral health changes during pregnancy but unfortunately the lack of knowledge and awareness about the same and its effect on the pregnancy outcome is still very much prevalent. Further an unfavourable attitude in general for seeking care for dental problems adds to this. The most common dental problems in pregnancy are gingivitis and periodontitis. ${ }^{9}$ There are some other instances also, in which there is requirement of special attention by dental professionals. Moreover, it should be kept in mind that the deleterious effect of these conditions not only affects the mother but also has impact on the new born, if proper attention is not paid to the situation. ${ }^{10}$

\section{GINGIVITIS}

Inflammation of gingiva or gingivitis is the most commonly seen change in oral cavity during pregnancy which accounts for about 60-75 per cent cases. ${ }^{11}$ It is more likely to occur during first trimester. In cases where the gingivitis already pre-exists, there is significant exacerbation of the situation. ${ }^{12}$ This occurs primarily due to alteration in levels of estrogen and progesterone and changes in oral flora and decreased immune response. Studies reveal that the estrogen 
levels may increase to as much as 30 times the normal levels and the progesterone may go up to 10 times. This in turn leads to greater vascular permeability and increased flow of the crevicular flow. The gingiva become red, edematous, bleeds and is tender on palpation from second trimester with peak level at about eight months. ${ }^{13}$ Based on the severity of gingivitis, patient may be advised for oral hygiene measures which include tooth brush and flossing for mild cases and professional scaling and root planning for cases which present with more severe form .

\section{PYOGENIC GRANULOMA (PREGNANCY TUMOR)}

Gingivitis may progress to become pyogenic granuloma. It is a smooth, lobulated exophytic lesion with sessile or pedunculated base. Most commonly present over the labial surface of papilla. It is present in about $0.2-0.5 \%$ of pregnant female starting as a benign rapidly growing lesion in first and second trimester. Local irritation factors like plaque are responsible for this granuloma which appears like a tumor like growth. Small lesions respond well to local debridement, chlorhexidine rinses, and other oral hygiene aids where as large lesions require deep excision. ${ }^{14}$

\section{PERIODONTITIS}

Periodontitis is the destructive inflammation of periodontium. According to different reports, the prevalence is ranged from $30 \%$ to $100 \% .{ }^{8}$ The periodontium is infiltrated by bacteria which leads to chronic inflammation. This process in turn leads to infection and destruction and breakdown of the periodontium, pocket formation and eventual loosening of the tooth. The increased levels of estrogen leads to decrease in the keratinisation of the periodontal tissues and hence and the epithelial barrier's efficiency is compromised. ${ }^{15}$ This process can induce recurrent bacteraemia, which indirectly triggers the hepatic acute phase response, resulting in production of cytokines, prostaglandins (PGE2), and interleukins (IL-6 and IL-8), all of these affects the pregnancy. ${ }^{16}$ Other association with adverse pregnancy outcome and dental problem is that periodontal diseases cause abnormal immunological changes which may also result in various complications during pregnancy. ${ }^{17}$ Various studies conducted worldwide have shown that there is some association of periodontitis with adverse pregnancy outcomes. ${ }^{18}$ There is sufficient evidence from a number of studies that if a woman is being treated for her periodontal disease by root planning and scaling during pregnancy, the chances of having a pre-term birth is significantly reduced. ${ }^{19,20,21}$. Besides pre-term birth, there is evidence of unfavourable effects of periodontal disease on pregnancy outcome. These includes miscarriage, still births, intra uterine growth retardation, pregnancy epulis, gestational diabetes and pre-eclampsia. ${ }^{22}$ In some reports, there is an evidence of association between dental infections and pulmonary infections. ${ }^{23}$ Various pathogens found in periodontitis have also found to have significant association with systemic diseases like diabetes mellitus, respiratory conditions, osteoporosis 
and cardiovascular diseases. ${ }^{24}$ It has been reported that during pregnancy, there is some aerobic to anaerobic change seen in the oral flora which may trigger inflammatory mediators which cause disturbances in lamina dura leading to tooth mobility. ${ }^{25}$

\section{TOOTH EROSION}

Pregnancy is accompanied by morning sickness in the first trimester. This invariably leads to nausea and vomiting. Acid reflux is increased due to a lax oesophageal sphincter and upward pressure from the gravid uterus. Patients with hyperemesis gravidarum causes enamel erosions. ${ }^{26}$ Changed dietary habits and frequency of foods may lead to increased chances of decay.

Ptyalism is also seen in case of pregnancy which can be reduced by consuming complex carbohydrates such as wheat breads, rice, oats, and corn. Changes in diet and lifestyle along 'with the use of anti-emetics, antacids or both can be used to manage the acid exposure. This can effectively be controlled with the use of a solution containing sodium bicarbonate which neutralizes acid and prevents damage. ${ }^{27}$ Pregnant women should be advised to brush every time after they vomit with the tooth brush of soft bristles so as to reduce the risk of enamel erosion. Fluoride mouth wash and fluoridated tooth pastes can also be used to protect eroded or sensitive teeth. ${ }^{28}$

\section{DENTAL CARIES}

Pregnant women are more prone to dental caries due to increased appetite and more frequent consumption of cariogenic foods. ${ }^{29}$ Recurrent vomiting becomes common in pregnancy which enhances acidic environment leading to progress of carious pathogens and increase in demineralization making teeth prone to caries ${ }^{30}$ Untreated carious lesions increase the incidence of abscess and cellulitis. ${ }^{31}$ Good oral hygiene practices by the expectant mother can be very instrumental in prevention of early childhood caries in children. ${ }^{32}$.It is seen that children whose mothers have active carious lesions in the oral cavity are more likely to transmit to it to them. ${ }^{33}$ This is caused by the transmission of the cariogenic bacteria Streptococcus mutans. This bacterial transmission may lead to inception of dental caries in the infant's oral cavity. ${ }^{34}$ Pregnant patients should be advised to brush twice daily with fluoridated toothpaste and they should also be advised to decrease the sugar intake.

\section{MANAGEMENT OF DENTAL HEALTH IN PREGNANT PATIENTS}

Management of dental health is a complex process and requires good knowledge of the various physiological changes taking place during pregnancy. Till few years back, there was some reluctance to provide definite dental treatments during pregnancy for risk to the mother and foetus and they were postponed for the post-partum period. This was because of absence of distinct guidelines by the health and regulatory bodies but now with more and more health bodies coming up with clear set of guidelines and better understanding of the needs of the 
dental health concerns and management strategies in pregnant patients have instilled greater confidence in the treating dental professionals. Collaboration between the treating obstetrician, medical personnel, nursing staff and dental professionals would stand in high interest of holistic management of the patient. ${ }^{35}$

High risk patients (such as patients with pregnancy induced hypertension, gestational diabetes, history of premature labour or complicated pregnancies) can be identified by taking detailed medical history. Careful monitoring of baseline blood pressure, pulse rate and respiratory rate are mandatory and if there are any changes then obstetrician should be notified immediately. ${ }^{36}$ Dental treatment of any kind should be avoided during first trimester so that there is no harm during organogenesis of foetus. Ideally, dental treatment should be done in the beginning of second trimester as there is no risk of teratogenesis, nausea and vomiting. Also, the size of uterus is not that large yet to cause any discomfort. ${ }^{37}$ Moreover, it is important to treat all dental problems during second trimester to facilitate intubations during labour if necessary. ${ }^{38}$

During third trimester of pregnancy, the patient's head should always be placed higher than her feet on dental chair and if required, left uterine displacement should be done so that uterus is moved away from the inferior vena cava. ${ }^{39}$

Dental radiography may be performed at any time in pregnancy for acute diagnostic purposes. Dentist must follow all proper radiological practices such as using radiation active apron with a thyroid collar, high speed films as well as following proper procedures to take radiograph. ${ }^{40}$ Regarding medication, acetaminophen is the most common and safest drug used in pregnancy. Adverse effect of acetaminophen is hepatotoxicity and it may also lead to toxicity if dose exceeds above 4 gms per day. ${ }^{41}$ Most antibiotics prescribed belongs to category B of FDA classification. The safest drug during pregnancy is paracetamol as it is not teratogenic.

Ciprofloxacin is used to treat periodontal problems but its use is restricted as it may cause arthropathy and other adverse effects on cartilage development. ${ }^{42}$ Metronidazole should be used with caution during first trimester as it is thought to be teratogenic but recent studies give no relevant data. ${ }^{43}$ Topical application fluoride may cause nausea, so fluoride varnish may be used. ${ }^{44}$ The long term exposure to $\mathrm{N}_{2} \mathrm{O}$ may lead to spontaneous abortion and birth defects. ${ }^{44}$ Intravenous administration of local anaesthetic with epinephrine may cause insufficiency of uteroplacental blood flow. However, 1:100,000 epinephrine concentration is safe in healthy patients.$^{45}$

\section{Collaborative Approach for Management of Oral Health In Pregnancy}

It is well documented that there is a serious lag in providing and obtaining optimum dental care during the pregnancy. A number of surveys have been done to find out the reasons for the same. This practice is more evident in developing nations though. ${ }^{46}$ Oral health is neglected on a whole and pregnancy further adds to a lot of myths associated with seeking oral care and acts 
as a barrier. Another reason for not attending to the dental needs is the strong lack of education and awareness about the link between the oral health and systemic health and specially as discussed earlier, the effect of oral health on the outcome of pregnancy. A treating obstetrician and medical professional are the first and mandatory contact for every pregnant woman and can be very instrumental in improving awareness of oral health concerns and seeking oral care in collaboration with the dental professional. Every pregnant patient should be referred to the dental professional for thorough dental check-up and management of the any dental problems. In fact, the patients may be referred to a dental professional for a routine dental check -up as soon as they start to plan having a child so that their dental needs if any are taken care of at the right time. Oral health screening and comprehensive examination can become an integral part of prenatal health check-ups. ${ }^{47,48}$ Knowledge, awareness and education about oral health and its timely management and specifically during pregnancy can benefit the patients at large. Patients can be counselled to get the treatment on time. The reception offices and waiting rooms of both the medical and dental professionals should host relevant leaflets, brochures, educational videos and posters about the importance of oral health during pregnancy. ${ }^{49,50}$ This will help in community spread of awareness about the importance of oral health during pregnancy. There is a strong need for development of patient outreach and education programmes and clinical resources to integrate oral health with overall health during pregnancy into practice. It is absolutely recommended to integrate prenatal preventive oral health care into practice workflow. In developing nation like ours, this kind of initiative and collaborative approach will also help to curb lot of myths related to pregnancy which are prevalent in our social set ups and deter a pregnant women form seeking care for her dental needs.

\section{CONCLUSION}

Mouth is the mirror of various systemic changes such as pregnancy, puberty and menarche. The oral health provider should be aware of the plethora of changes in the oral cavity. Appropriate diagnosis and management of oral manifestations in a pregnancy is essential as the consequences of poor oral health may have a lifelong impact. Oral health check-up and preventive treatment should be an integral part of the pre-natal health programme. Education and counselling of the patients must be done to apprise them about the importance of oral health and its effects on the outcome of pregnancy. An amalgamated approach to management of pregnant women for their dental health concerns by both a dental and medical professional can bring a favourable change which would hugely benefit the patients.

\section{REFERENCES}

1. Marla V, Srii R, Roy DK, Ajmera H. The Importance of oral health during pregnancy: A review. Medical Express 2018; 5:1-6. 
2. Patil S, Thakur R, Madhu K, Paul ST. Gadicherla P. Oral health coalition: knowledge, attitude, practice behaviours among gynaecologists and dental practitioners. J Int Oral Health. 2013; 5(1): 8-15.

3. Hemalatha V,Manigandan T,Sarumathi T, Nisha VA, Amudhan A. Dental consideration in pregnancy - a critical review on oral care. J Clin Diagn Res 2013:7(5):948-53.

4. Hom JM, Lee JY, Divaris K, Baker AD, Vann WF Jr. Oral health literacy and knowledge among patients who are pregnant for the first time. J Am Dent Assoc 2012; 143:972-80.

5. Ramos-Gomez F, Jue B, Bonta CY. Implementing an infant oral care program. J CA Dent Assoc. 2002; 30:752-61.

6. Silk H. Douglass AB, Douglas JM, Silk L. Oral health during pregnancy. Am J Public Health 2008; 77:1139-44.

7. Kumar J, Samelson R. Oral health care during pregnancy recommendations for oral health professionals. N Y State Dent J 2009; 75(6):29-33.

8. Gupta R, AcharyaAK.Oral health status and treatment needs among pregnant women of Raichur District, India: A population based cross-sectional study. Scientifica 2016: Article ID 9860.DOI:10.1155/2016/9860387.

9. Laine MA. Effect of pregnancy on periodontal and dental health.ActaOdontolScand.2002; 60(5):257-64.DOI:10.1080/00016350260248210.

10. Rainchuso L.Improving oral health outcomes from pregnancy through infancy. J Dent Hyg.2013; 87(6):330-5.

11. American Dental Association Council on Access, prevention and inter professional relations. Women's oral health issues. American Dental Association, 2006.

12. Hey Hadavii JH. Women's oral health issues: sex differences and clinical implications. Women's Health Prim Care.2002; 5(3):189-199.

13. Laine MA. Effect of pregnancy on periodontal and dental health. ActaOdontol Scand.2002; 60(5):257-64.DOI:10.1080/00016350260248210.

14. Tucker R.Periodontitis and pregnancy. J R Soc Prompt Health 2006; 126:24-7.

15. Slade GD, Ghezzi EM, Heiss G, Beck JD, Riche E, Offenbacher S. Relationship between periodontal disease and Creactive protein among adults in the atherosclerosis risk in communities study. Arch Intern Med. 2003; 163(10):1172-9.

16. Dörtbudak O, Eberhardt R, Ulm M, Persson GR. Periodontitis, a marker of risk in pregnancy for preterm birth. J Clin Periodontol. 2005; 32(1):45-52. 
17. George A, Dahlen HG, Blinkhorn A, Ajwani S, Bhole S Ellis S, et al. Measuring oral health during pregnancy: sensitivity and specificity of a maternal oral screening (MOS) tool.BMC Pregnancy Childbirth. 2016; 16(1):347.

18. Han YW. Oral Health and Adverse Pregnancy Outcomes - What's Next? J Dent Res. 2011; 90(3):289-93. DOI: 10.1177/0022034510381905.

19. Shanthi V, Vanka A, Bhambal A, Saxena V, Saxena S, Kumar SS. Association of pregnant women periodontal status to preterm and low birth weight babies: A systematic review and evidence based review. Dental Res J. 2012;9(4):368-80.

20. Polyzos NP, Polyzos IP, Mauri D, Tzioras S, Tsappi M, Cortinovis I, et al. of periodontal disease treatment during pregnancy on preterm birth incidence: a meta analysis of randomized trials. Am J Obstet Gynecol. 2009 Mar; 200(3):225-32. doi: 10.1016/j.ajog.2008.09.020.

21. Vettore MV, Lamarca GA, Leão AT, Thomaz FB, Aubrey S, Leal MC. Periodontal infection and adverse pregnancy outcomes: a systematic review of epidemiological studies. Cad Saude Publica 2006 Oct;22(10):2041-53. DOI: 10.1590/S0102311X2006001000010.

22. Shub A, Wong C, Jennings B, Swain JR, Newnham JP. Maternal periodontal disease and perinatal mortality. Aust NZ J Obstet Gynaecol.2009; 49:130-6. DOI:10.1111/j.1479-828X.2009.00953.x

23. Ameet M. M, Avneesh H. T, Babita R. P, Pramod P. M. The Relationship between Periodontitis and Systemic Diseases- hype or hope? Journal of Clinical and Diagnostic Research: JCDR. 2013; 7(4):758-62. DOI:10.7860/JCDR/2013/4500.290624.

24. Winning L, Linden GJ. Periodontitis and systemic disease. Association or Causality? .Curr Oral Health Rep. 2017; 4(1): 1-7.

25. Miller WD. The human mouth as a focus of infection. Lancet. 1891; 138(3546):340-2

26. American Dental Association Council on Access, Prevention and Inter Professional Relations. Women's oral health issues. American Dental Association, 2006.

27. SJ.Ramirez B. Lazarchik DA. Vaczi MF, Richter JE. Dental erosion and acid reflux disease. Ann Intern Med 1995; 122:809-15.

28. Lewis CW, Milgrom P. Fluoride [published correction appears in Pediatr Rev. 2003; 24(12):429]. Pediatr Rev. 2003; 24(10):327-336.

29. Geum Joon Cho, So-youn Kim, Hoi Chang Lee, Ho Yeon Kim, Kyu-Min Lee, Sung Won Han \& Min-Jeong Oh. Association between dental caries and adverse pregnancy outcomes. Sci Rep 10, 5309 (2020). https://doi.org/10.1038/s41598-020-62306-2

30. Silk H. Douglass JM. Silk L. Oral health during pregnancy. Am Fam Physician 2008:77:1139-44. 
31. Giglio JA. Lanni SM, Laskin DM, Giglio NW. Oral health care for the pregnant patient. J Can Dent Assoc 2009:75:43-8.

32. American College of Obstetricians and Gynaecologists Women's Health Care Physicians Committee on Health Care for Underserved Women. Committee opinion no. 569: Oral health care during pregnancy and through the lifespan. Obstetrics and Gynaecology 2013; 122:417-22.

33. Berkowitz RJ. Acquisition and transmission of mutans streptococci. J Calif Dent Assoc. 2003; 31(2):135-138.

34. U.S. Department of Health and Human Services, National Institute of Dental and Craniofacial Research. Oral Health in America: A Report of the Surgeon General. NIH publication no. 00-4713. Rockville, Md.: U.S. Public Health Service, Dept. of Health and Human Services; 2000.

35. Kumar J and Samelson R. Oral health care during pregnancy recommendations for oral health professionals. New York State Dent J. 2009; 75(6):29-33.

36. Little JW, Falace DA, Miller CS, Rhodus NL. Dental Management of the Medically Compromised Patient. 7th ed. St. Louis: CV Mosby; 2008. p. 268-278, 456.

37. Thornburg KL, Jacobson SL, Giraud GD, Morton MJ. Hemodynamic changes in pregnancy. Semin Perinatol 2000; 24:11-4.

38. Mangskau KA, Arrindell B. Pregnancy and oral health: Utilization of the oral health care system by pregnant women in North Dakota. Northwest Dent 1996; 75:23-8.

39. New York State Department of Health (NYSDH). Oral Health Care During Pregnancy and Early Childhood, Guidelines; 2006.

40. Cnattingius S, Lambe M. Trends in smoking and overweight during pregnancy: Prevalence, risks of pregnancy complications, and adverse pregnancy outcomes. Semin Perinatol 2002; 26:286-95.

41. Grahme Smith DG, Aronson JK. Oxford textbook of clinical pharmacology and drug therapy, 1992.ISBN 0-19-850944-8;

42. Bomford J, Ledger J, Okeeffe B, Reiter C, Ciprofloxacin use during the pregnancy. Drugs 1993; 45:461-62.

43. American Academy of Pediatric Dentistry (AAPD). Guideline on Oral Health Care for the Pregnant Adolescent. Council on Clinical Affairs Committee on the Adolescent. Chicago: AAPD; 2007.

44. US Food and Drug Administration. Labeling and prescription drug advertising: Content and format for labeling for human prescription drugs. Fed Regist 1979; 44:434-67.

45. Haas DA, Pynn BR, Sands TD. Drug use for the pregnant or lactating patient. Gen Dent 2000; 48:54-60. 
46. Neha Gupta, Manisha Chhetry: Knowledge and Practices of Pregnant Women regarding Oral Health in a Tertiary Care Hospital in Nepal. JNMA J Nepal Med Assoc, May-Jun 2019;57(217):184-188.

47. Erin Hartnett, Judith Haber, Barbara Krainovich-Miller, Abigail Bella, Anna Vasilyeva, and Julia Lange Kessler Oral Health in Pregnancy. JOGNN, 45, 565-573; 2016

48. Megan K. Kloetzel, Colleen E. Huebner, Peter Milgrom: Referrals for Dental Care During Pregnancy.J Midwifery Women’s Health. 2011 March; 56(2): 110-117

49. .New York State Department of Health. Oral health care during pregnancy and early childhood: Practice Guidelines. New York State Department of Health; 2006.

50. Gaffield ML, Gilbert BJ, Malvitz DM, Romaguera R. Oral health during pregnancy: an analysis of information collected by the pregnancy risk assessment monitoring system. J Am Dent Assoc. 2001; 132:1009-16. [PubMed: 11480627]

\section{BJMHR is}

- Peer reviewed

- Monthly

- Rapid publication

- Submit your next manuscript at editor@bjmhr.com 